\title{
Research Paper \\ Effect of Eight Weeks of 11+ Training on Functional Movement Screening Test Scores in Female Futsal Players
}

\author{
*Akram Kiani Sheikhabadi ${ }^{1}$ (), Reza Mahdavinejad², Nader Rahnma² ${ }^{\mathbb{C}}$
}

1. Department of Sport Pathology and Corrective Movements, Faculty of Physical Education, Khorasgan Branch, Islamic Azad University, Isfahan, Iran 2. Department of Sport Pathology and Corrective Movements, Faculty of Sport Science, Isfahan University, Isfahan, Iran.

\begin{tabular}{|l|l|}
$\begin{array}{c}\text { Use your device to scan } \\
\text { and read the article online }\end{array}$ & $\begin{array}{l}\text { Cftation: Kiani Sheikhabadi A, Mahdavinejad R, Rahnma N. [Effect of Eight Weeks of 11+ Training on Functional Move- } \\
\text { ment Screening Test Scores in Female Futsal Players (Persian)]. Journal of Sport Biomechanics. 2020; 6(1):2-11. https://doi. } \\
\text { org/10.32598/biomechanics.6.1.2 }\end{array}$ \\
doi https://doi.org/10.32598/biomechanics.6.1.2
\end{tabular}

\section{(c) (i)}

Article Info:

Received: 12 Mar 2020

Accepted: 17 May 2020

Available Online: 01 Jun 2020

Key words:

Functional move-

ment screening, 11+

exercises, Futsal,

Injury prevention

\section{A B STRACT}

Objective The purpose of this study is to determine the effect of an 8-week 11+ exercise program on seven test scores of Functional Movement Screening (FMS) in female futsal players.

Methods This quasi-experimental study was conducted on 48 female futsal players who were selected using purposive and convenience sampling methods and randomly divided into two groups of exercise $(n=36$; Mean $\pm S D$ age $=23.3 \pm 2.8$ years; Mean $\pm S D$ height $=164.94 \pm 4.93 \mathrm{~cm}$; Mean $\pm S D$ weight $=63.58 \pm 6.35$ $\mathrm{kg}$ ) and control $(\mathrm{n}=12$; Mean \pm SD age $=23.08 \pm 2.74$ years; Mean \pm SD height $=165.42 \pm 5.08 \mathrm{~cm}$; Mean $\pm S D$ weight $=62.83 \pm 6.10 \mathrm{~kg}$ ). Seven FMS tests were carried out before and after implementation of the $11+$ exercise protocol for 8 weeks, 3 sessions per week each for 20 minutes. For analyzing the data, repeatedmeasures ANOVA was used considering a significant level of $P \leq 0.05$.

Results The $11+$ exercise program had a significant interaction effect on the scores of deep squat $\left(F_{(1.46)}=6.07 ; P=0.01\right)$, hurdle step $\left(F_{(1.46)}=4.98 ; P=0.03\right)$, and in-line lunge $\left(F_{(1.46)}=11.63 ; P=0.001\right)$ in subjects, but had no significant effect on their shoulder mobility $\left(F_{(1.46)}=2.19 ; P=0.14\right)$, active straight-leg raise $\left(F_{(1.46)}=2.003 ; P=0.16\right)$, trunk stability push-up $\left(F_{(1.46)}=1.09 ; P=0.30\right)$, and rotary stability $\left(F_{(1.46)}=0.20 ; P=0.20\right)$. Conclusion It seems that the 11+ exercise program was effective in improving the FMS scores of female futsal players. It can be used by coaches and corrective exercises experts as a training method for injury prevention before training in female futsal players.

\section{Extended Abstract}

\section{Introduction}

$\mathbf{F}$

ollowing the encouragement of the general public in a community to increase participation in sports activities, unfortunately, the number of people at risk of injury has increased [1]. One of the im- which can lead to productivity and reduction of medical expenses and frequent absences from work [2]. Epidemiological studies have shown that one sixth of all injuries observed by physicians occur during sports activities and the rate of injuries has increased following an increase in participation in sports activities [3], where the lower limbs including knee and ankle joints have had the most injuries [4]. Screening is performed to prevent injury as well as to improve implementation strategies [8].

portant priorities of the society is to have healthy and energetic youth and capable and efficient human resources,

\section{* Corresponding Author:}

\section{Akram Kiani Sheikhabadi, MSc.}

Address: Department of Sport Pathology and Corrective Movements, Faculty of Physical Education, Khorasgan Branch, Islamic Azad University, Isfahan, Iran.

Tel: +98 (913) 5491618

E-mail: akramkiani.vah@gmail.com 
Functional Movement Screen (FMS) method includes seven movement tests that have the ability to identify limitations and changes in normal movement patterns. These tests are designed to interact between postural chain mobility and the stability required to perform functional and essential movements [9]. One of the core stability trainings is the $11+$ exercise program, which include strength exercises and core stability exercises suitable for muscle strength and dynamic and static balance at three levels, which have recently been considered by FIFA [12]. The aim of this study was to investigate the effect of an 8-week 11+ exercise program on the FMS scores in female futsal players.

\section{Methods}

This is a quasi-experimental study. Study population consists of all female futsal players in Isfahan, Iran. Of these, 48 were selected using a convenience sampling method and assigned into two groups of exercise and control. First, a demographic form was completed by the subjects, which includes personal information such as height, weight, age, playing history, activity per week, and history of injury or illness. After obtaining written consent, all subjects meeting entry criteria (no history of injury or surgery in the past 6 months) were evaluated by FMS tests before and after intervention. In order to evaluate the scores of FMS, a special kit consisting of a FMS board with 56 inch (calibrated 43 inch) length, 6 inch width, and 2 inch height; graded long rod with a length of 48 inches, two short rods with a length of 25 inches, an elastic band, and data collection forms were used [7]. The FMS tests included: Deep Squat, hurdle step, in-line lunge, active straight-leg raise, trunk stability push-up, quadruped rotary stability, and shoulder mobility. The protocol of $11+$ exercise program had three parts according to the guidelines of FIFA Medical Assessment and Research Center.

\section{Results}

The demographic characteristics of participants are presented in Table 1. According to the results of independent t-test, the 2 groups of control and exercise were homogeneous $(\mathrm{P}>0.05)$. In examining the effect of group on deep squat score, within-group effect $\left(\mathrm{P}=0.07 ; \mathrm{F}_{(1.46)}=3.3\right)$ and between-group effect $(\mathrm{P}=0.9$; $\left.\mathrm{F}_{(1.46)}=0.006\right)$ were non-significant but the interaction effect was significant $\left(\mathrm{P}=0.01 ; \mathrm{F}_{(1.46)}=6.07\right)$ (Figure 1). Regarding the effect of group on hurdle step score, within-group $\left(\mathrm{P}=0.03 ; \mathrm{F}_{(1.46)}=4.98\right)$ and interaction effects $\left(\mathrm{P}=0.03\right.$ and $\left.\mathrm{F}_{(1.46)}=4.98\right)$ were significant but between-group effect $\left(\mathrm{P}=0.04 ; \mathrm{F}_{(1.46)}=0.82\right)$ was not significant (Figure 1).

Regarding the effect of group on in-line lunge score, within-group $\left(\mathrm{P}=0.62 ; \mathrm{F}_{(1.46)}=0.23\right)$ and between-group effects $\left(\mathrm{P}=0.84 ; \mathrm{F}_{(1.46)}=0.03\right)$ were insignificant, but the interaction effect $\left(\mathrm{P}=0.001 ; \mathrm{F}_{(1.46)}=11.61\right)$ was significant (Figure 1). Regarding the group effect on the shoulder mobility score, due to the non-significant interaction effect, the null hypothesis was confirmed and the research hypothesis was rejected (Figure 1). Regarding the effect of group on active straight-leg raise score, within-group effect $\left(\mathrm{P}=0.02 ; \mathrm{F}_{(1.46)}=5.56\right)$ was significant but between-group effect $\left(\mathrm{P}=0.70 ; \mathrm{F}_{(1.46)}=0.15\right)$ and interaction effect $\left(\mathrm{P}=0.16 ; \mathrm{F}_{(1.46)}=2.003\right)$ were not significant (Figure 1).

Regarding the effect of group on trunk stability pushup score, within-group effect $\left(\mathrm{P}=0.005 ; \mathrm{F}_{(1.46)}=8.57\right)$ was also significant, and between-group effect $(\mathrm{P}=0.17$; $\left.\mathrm{F}_{1.46)}=1.88\right)$ and interaction effect $\left(\mathrm{P}=0.30 ; \mathrm{F}_{(1.46)}=1.09\right)$ were not significant (Figure 1). Regarding the effect of group on rotary stability score, within-group effect $\left(\mathrm{P}=0.36 ; \mathrm{F}_{(1.46)}=0.82\right)$, between-group effect $(\mathrm{P}=0.6$; $\left.\mathrm{F}_{(1.46)}=0.28\right)$ and interaction effect $\left(\mathrm{P}=0.65 ; \mathrm{F}_{(1.46)}=0.20\right)$ were not significant (Figure 1). The between-group differences in FMS scores were the same between pretest and posttest phases $(\mathrm{P}>0.05)$.

Table 1. Demographic characteristics of participants

\begin{tabular}{cccccc}
\hline Variable & & Mean \pm SD & & T & P \\
\cline { 2 - 5 } & Exercise & Control & & 0.95 & 0.29 \\
\hline Age $(\mathrm{Y})$ & $23.2 \pm 3.8$ & $23.2 \pm 08.74$ & 0.98 & 0.33 \\
Height $(\mathrm{cm})$ & $164.4 \pm 94.93$ & $165.5 \pm 42.08$ & 1.5 & 0.11 \\
\hline Weight $(\mathrm{kg})$ & $63.6 \pm 58.35$ & $62.6 \pm 83.10$ & & Journal of \\
\hline & & & Sport Biomechanics
\end{tabular}



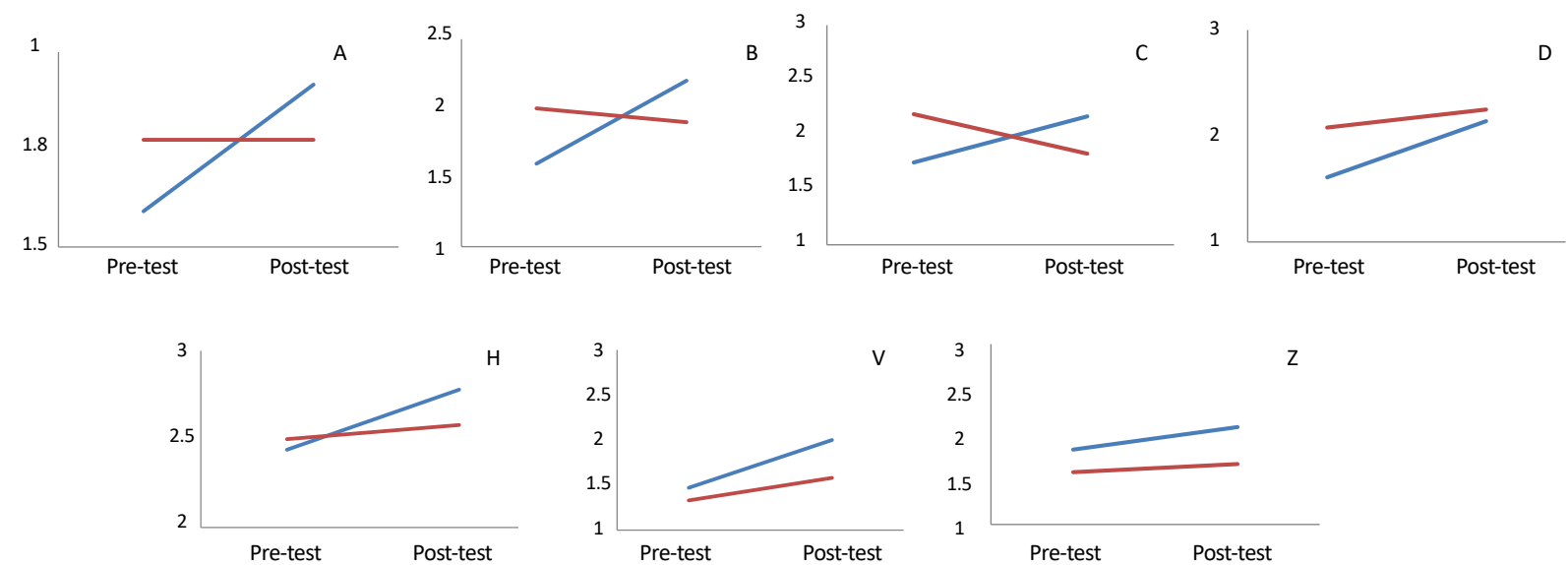

Journal of Sport Biomechanics

Figure 1. The effect of group on deep squat score

\section{Conclusion}

The 11+ exercise program can improve the scores of deep squat, hurdle step, and in-line lunge tests in FMS method in female futsal players, and can affect their overall FMS scores. It can be used as a modality by coaches, corrective exercise specialists, and physical education teachers to reduce and prevent sports injuries in female futsal players. It can identify poor movement patterns and improve performance.

\section{Ethical Considerations}

\section{Compliance with ethical guidelines}

Prior to study, a written informed consent was signed by all participants. They were free to leave the study at any time and were assured of the confidentiality of their information

\section{Funding}

This study was extracted from the MA. thesis of the first author, Department of Sport Pathology and Corrective Movements, Faculty of Physical Education, Khorasgan Branch, Islamic Azad University.

\section{Authors' contributions}

Writing final draft of the manuscript, data analysis, data mining: Akram Kiani Sheikhabadi; Data monitoring, resutls correction: Reza Mahdavinejad; Supervision: Nader Rahnma.

\section{Conflicts of interest}

The authors declared no conflict of interest.

\section{Acknowledgements}

The authors would like to thank the Deputy for Research of Islamic Azad University of Khorasgan Branch in Isfahan and all participants for their valuable cooperation. 


\title{
تأثير هشت هفته تمرينات +1 (ا بر امتيازات أزمون غربالكرى عملكرد حركتى فوتساليستهاى زن
}

\author{
"اكرم كيانى شيخآبادى' ه. رضا مهدوىنزاد'، نادر رهنما' (1) \\ ا. كروه آسيبشناسى ورزشى و حركات اصلاحى، دانشكده تربيتبدنى، دانشعاه آزاد اسلامى خوراسكان، اصفهان، ايران.

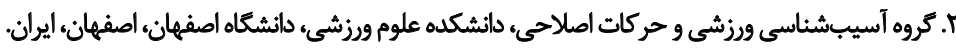

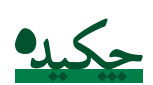

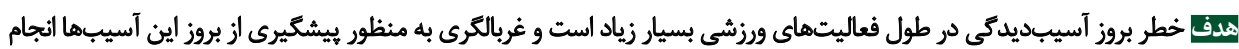

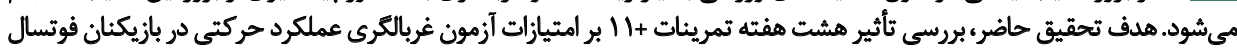

زئ بودا.

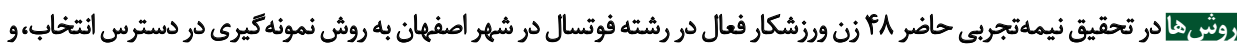

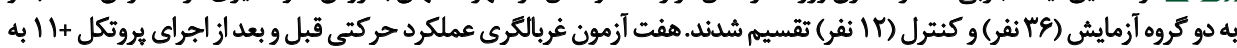

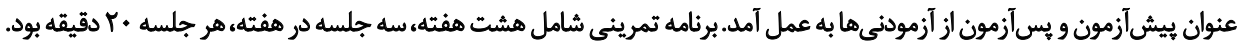

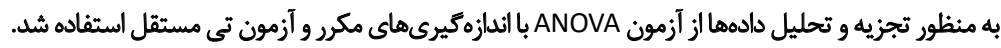

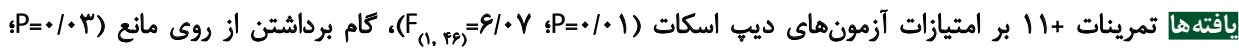

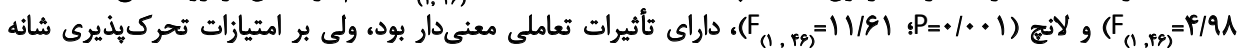

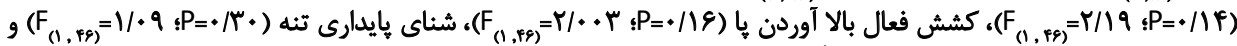
ثبات جرخشى (F) تأثير معنى

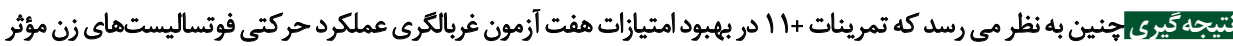

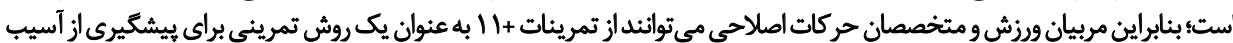

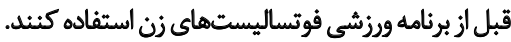

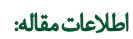

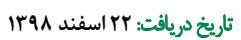

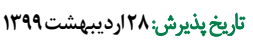
تاريخ انتشار: rاخرداد

كليدوازوها: آزمون غربالكرى عملكرد حركتي، تمرينات +11ا،

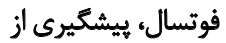
آسيب

يا بيشترين آسيبها را به خود اختصاص داده است [بأ] مسائل

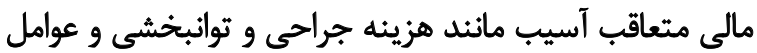

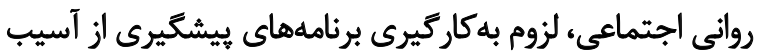

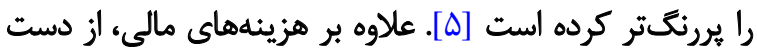

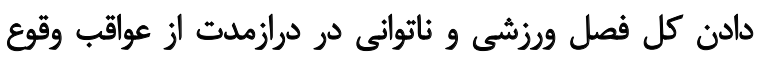

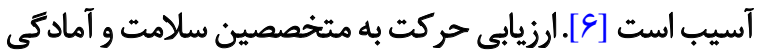

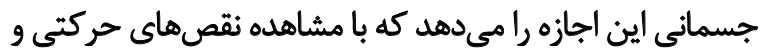

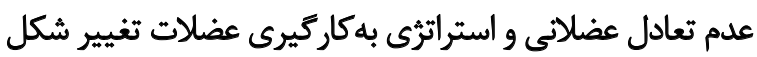

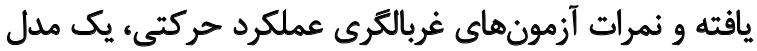

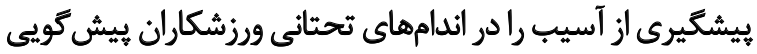

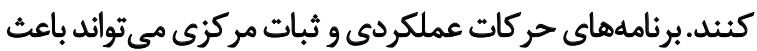

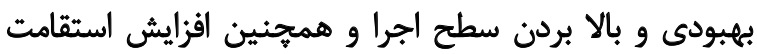

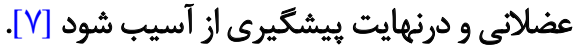

غربالكرى به منظور بيشعيرى از آسيب و همجنين ارتقاى

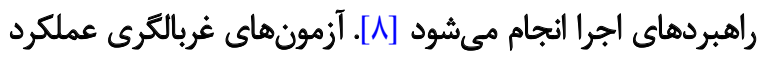

به دنبال تشويق و ترغيب عموم افراد يك جامعه به افزايش

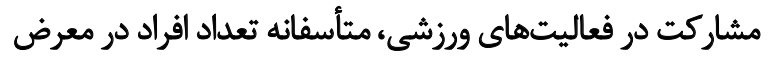

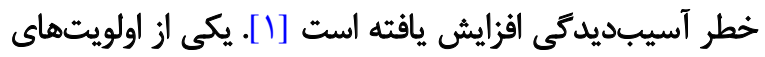

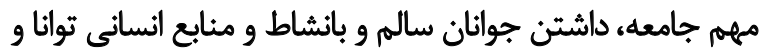

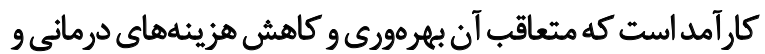

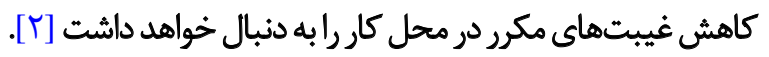

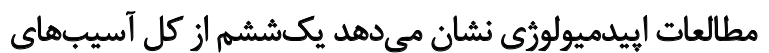

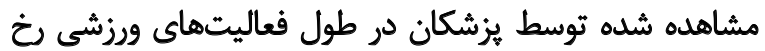

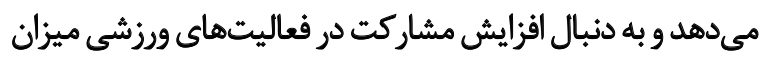

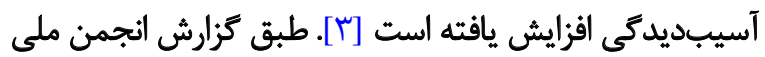

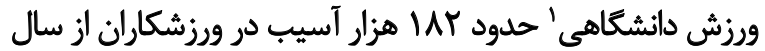

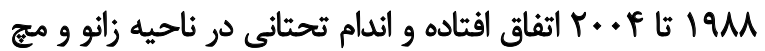

1. National Collegiate Athletic Association 

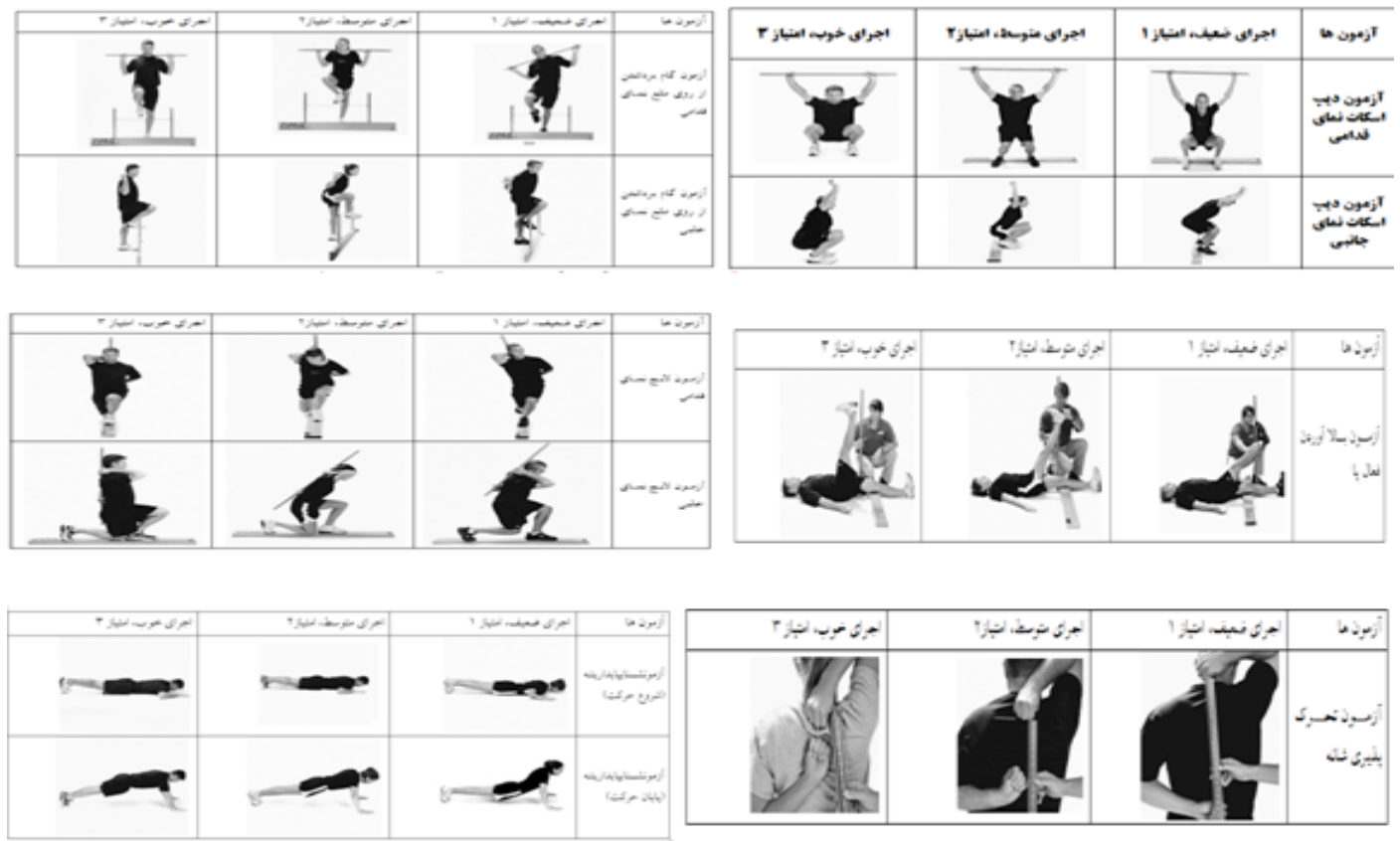

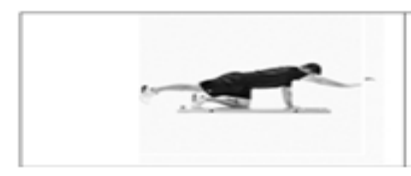

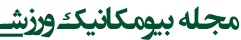

غربالكرى عملكرد حركتى را نيز به عنوان يك ابزار معتبر به كار

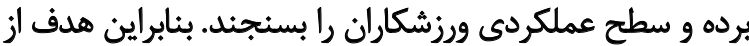

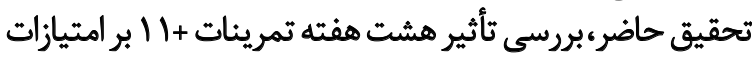
آزمونهاى غربالكرى عملكرد حركتى در بازيكنان فوتسال دختر

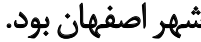

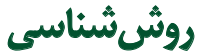

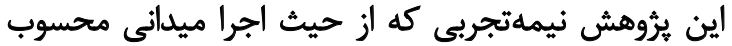

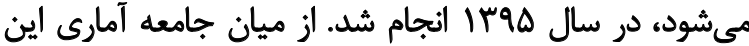

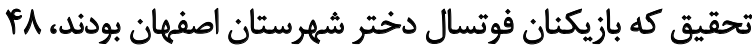

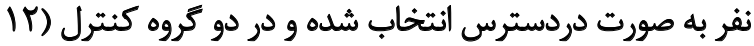

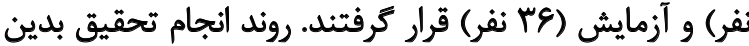

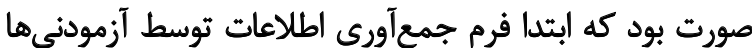

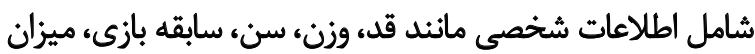

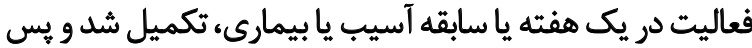

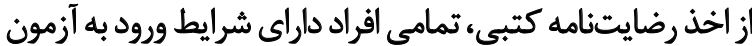

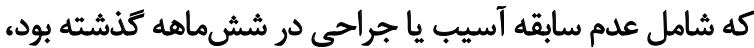

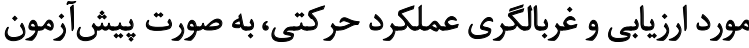

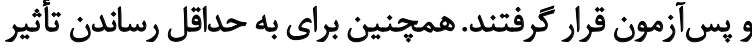

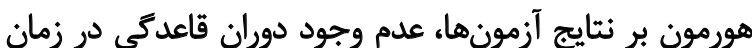
اجراى آزمون مدنظر قرار كرفت.

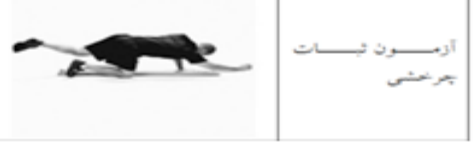

تصوير ا. الكوهاى حركتى هفت آزمون
حركتى (FMS) شامل هفت آزمون حركتى است كه داراى قابليت

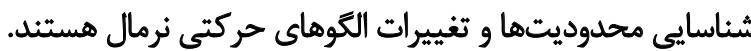

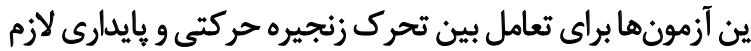

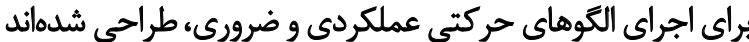

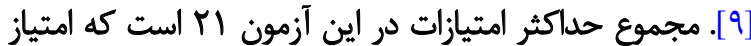

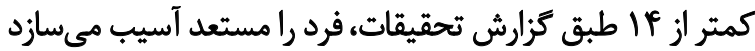

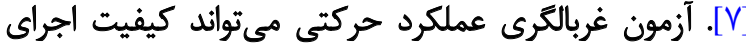

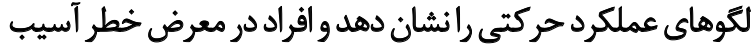

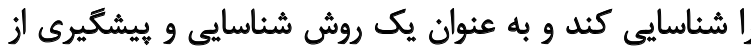

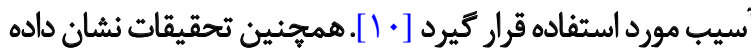

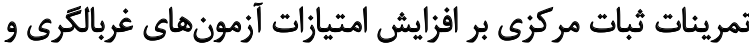

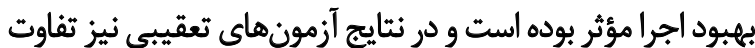

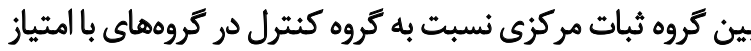

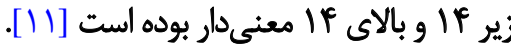
از جمله تمرينات ثبات مركزى، تمرينات +11 است، كه شامل

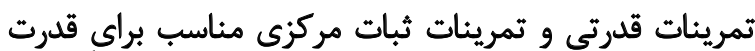

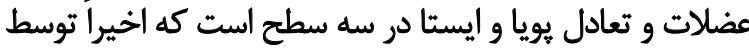

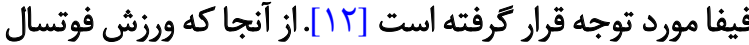

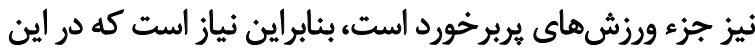

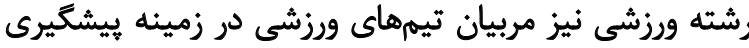

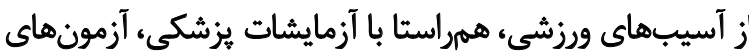


جدول ا. سه بخش يروتكل تمرينى +11 يا برنامه جامع كرم كردن فيفا

\begin{tabular}{|c|c|c|}
\hline \multicolumn{3}{|c|}{ بخش اول يروتكل جامع +||(: تمرينات دويدنى، زمان: A دقيقد } \\
\hline سطح سه & 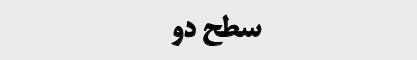 & سطع يكى \\
\hline ب. برخش داخلى ران & r. مخش ران به خارج & 1. اويلن به سمت جلو و مستقيم \\
\hline و دويلن سريع به جلو وعقب & هـ زدن شائهها به همديكر & ب. بهرخيلن دور يار تمرينى \\
\hline \multicolumn{3}{|c|}{ بخش دوم يروتكل جامع +1 (1: تمرينات قدرتى، يلايومتريك، تعادلى، زمان: + 1 دقيقه در سه سطح } \\
\hline سطح سه & 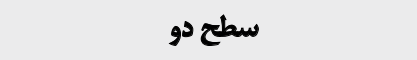 & سطح يك \\
\hline نيمكت با بلند كردن يك يا و نكاه داشتن & نيمكت با جابهجايع عتناوب باها & 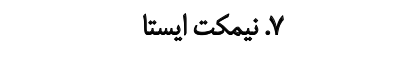 \\
\hline | يمكت جانبي با بلثد كردن ها & نيمكت جانبي همراه بالا و يايين بردن لكن & 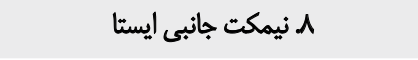 \\
\hline حركت همسترينَ در سطح ييشرفثه & رركت همسترينك در سطح متوسط & 9. حركت همسترينك سطح مبثدى \\
\hline ايسثادن روى يك با و بر هم زدن تعادل يار تمرينى & ايسثادن روى يك يا و يرثاب توب & •ا. ايستادن روى يك يا و نكله داشتن توبه \\
\hline اسكات روى يك يا & راه به شكل لانج & 1) \\
\hline يرش در جهات مختلف (يرش يابكس). & يرش جانيى & r آ يرش عمودى \\
\hline \multicolumn{3}{|c|}{ بخش سوم يروتكل جامع +1 (: تمرينات دويدنى، زمان: Y دقيقه } \\
\hline سطع سه & سطح دو & سطح يك \\
\hline 1ه حركات يرشى & أ ا. دويدن توأم با جهش & "ا. دويدن در عرض زمين \\
\hline
\end{tabular}

مجله بيومكانيك وزنث

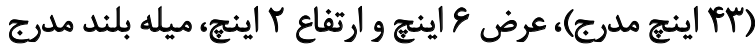

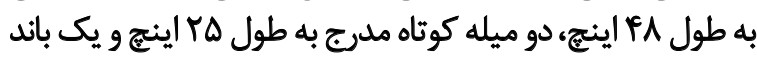

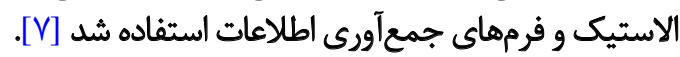
كليه الكوهاى حركتى آزمونهاى عملكرد حركتى (FMS)

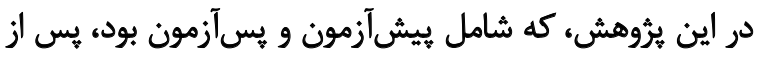

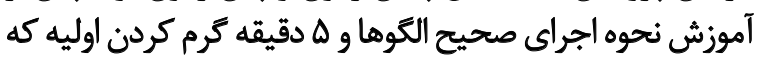

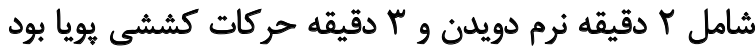

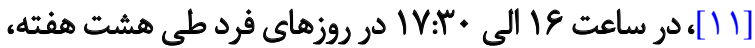

$$
\text { اجرا شد (تصوير شماره (). }
$$

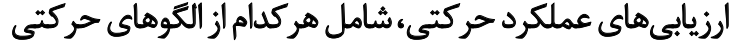

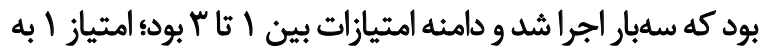

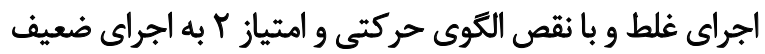

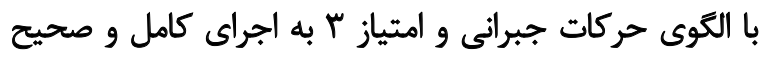

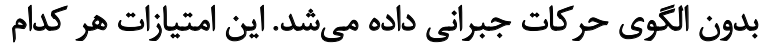

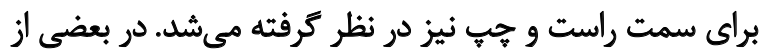

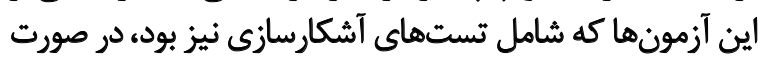

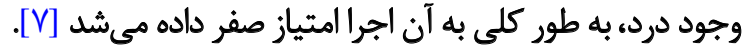
به منظور ارزيابى امتيازات آزمونهاى غربالكرى عملكرد

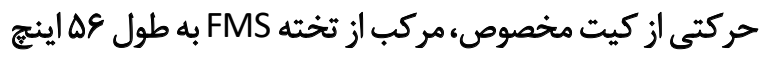




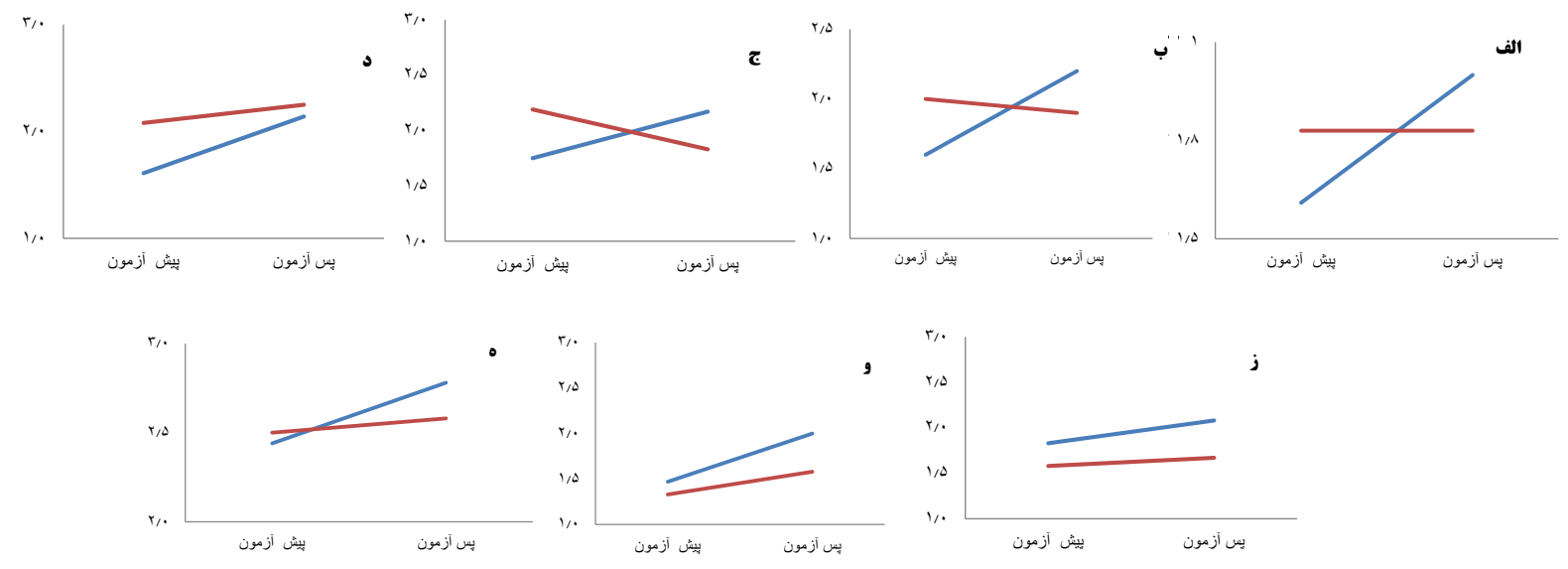

مجله بيومكانيك ورنشي

تصوير r. بررسى اثر كروهبندى بر امتياز آزمون ديب اسكات

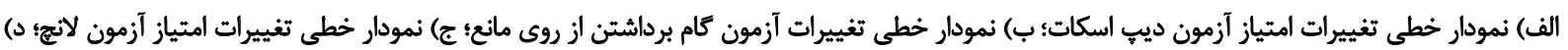

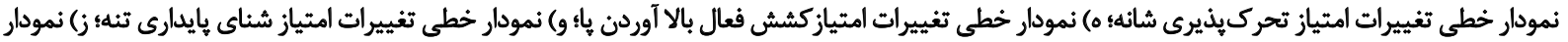
خطى تغييرات امتياز ثبات جرخشى (كروه آزمايش، خط آبى و مكروه كنترل، خط قرمز

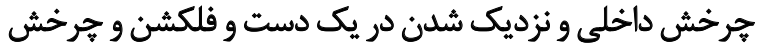

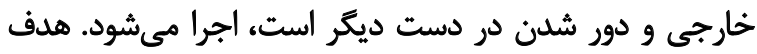

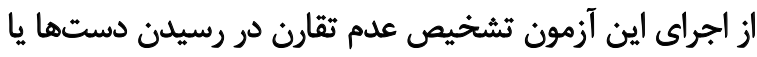

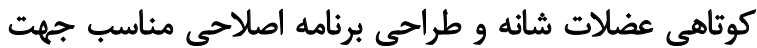

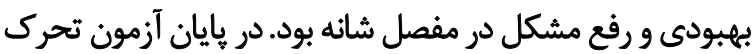

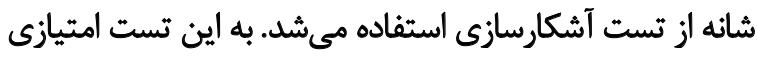

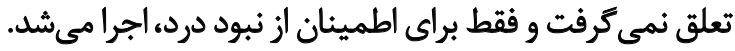

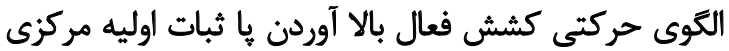

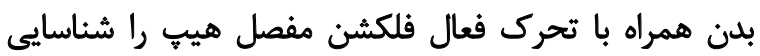

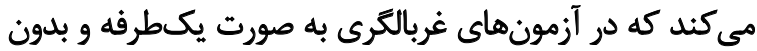

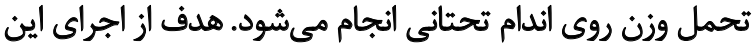

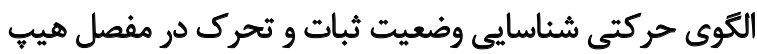

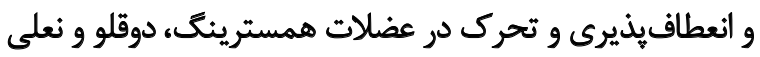

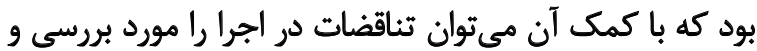

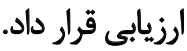

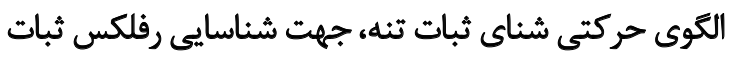

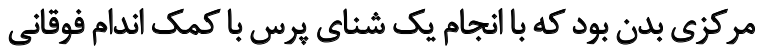

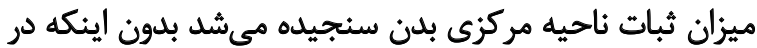

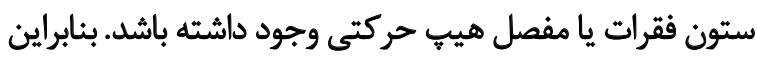

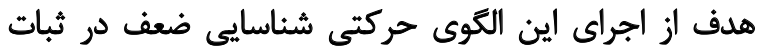

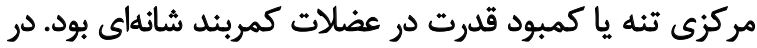

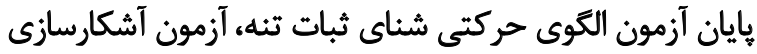

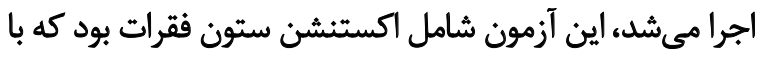
برس روى دستها انجام مي آشدا.

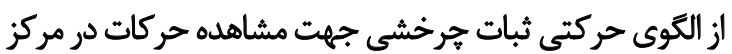

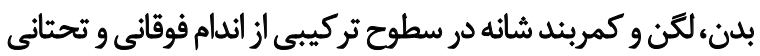

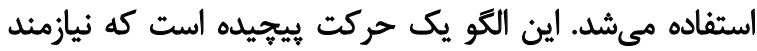

الكوى حركتى ديب اسكات، بخشى از حركات عملكردى است

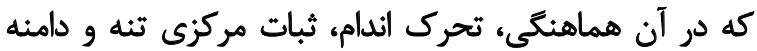

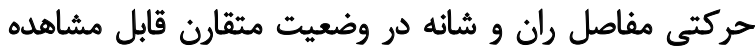

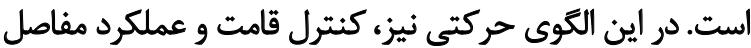

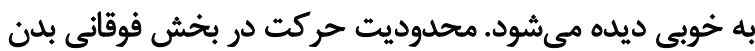

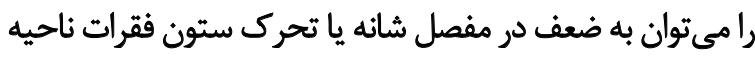

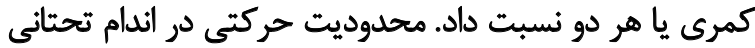

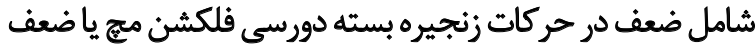

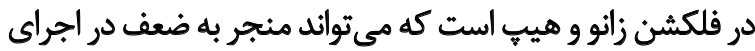

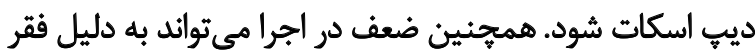

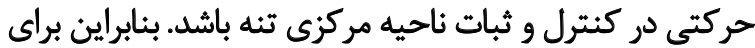

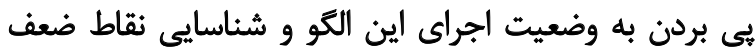
حركتى از اين آزمون استفاده شد.

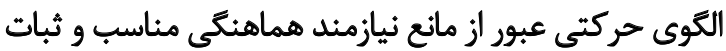

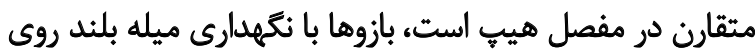

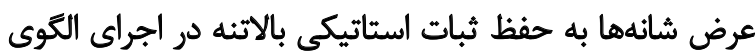

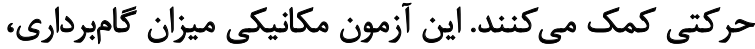

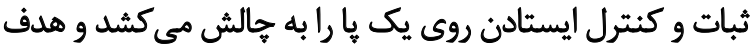

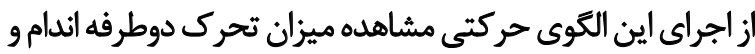

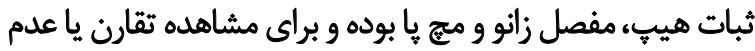

$$
\text { تقارن در بدن مناسب است. }
$$

الكوى حركتى لانج جزء حركات كاهش شتاب و تغيير مسير

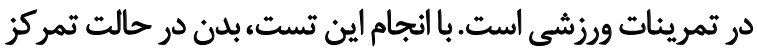

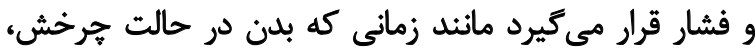

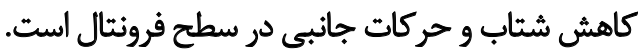

الكوى حركتى تحرك شانه، به صورت دوطرفه و به صورئ صورت نامتقارن در دامنه حركتى شانه كه تركيبى از اكستنشن و 


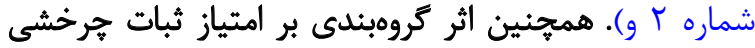

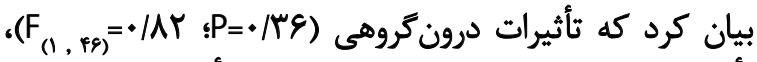

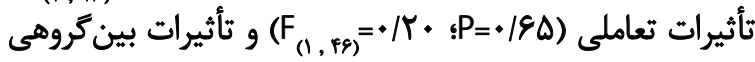

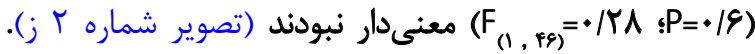

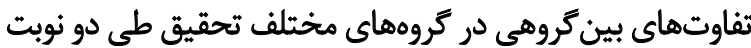
آزمون كيرى همسان بود (ه) (P> (P).

ث

هدف از بثروهش حاضر بررسى ثمرينات +11 بر المتيازيات

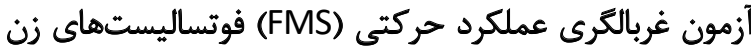

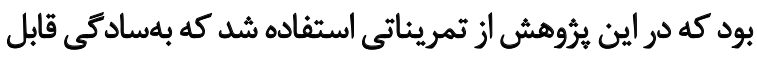
اجراو تكرار مجدد توسط ساير محققين است.

در تحقيق حاضر تأثير تمرينات +11 البر امتيازات ديب اسكات

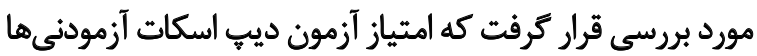

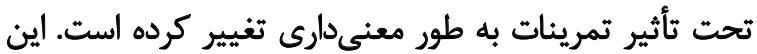

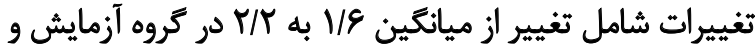

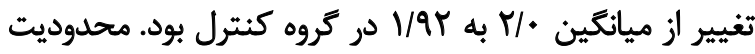

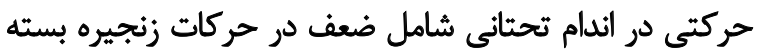

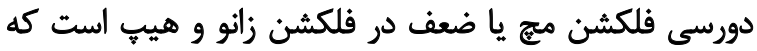

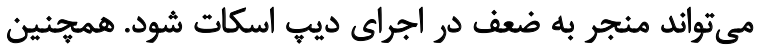

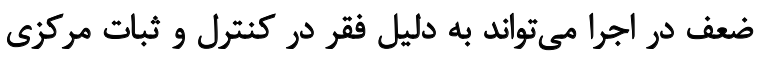

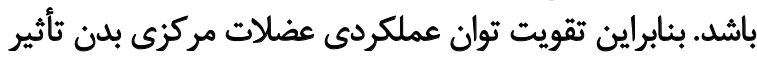

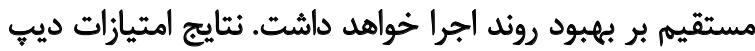

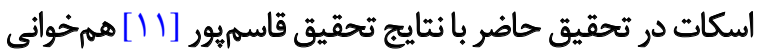

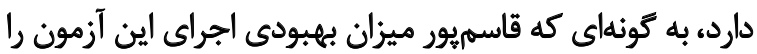

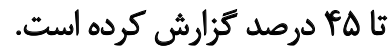

در اين تحقيق تأثير تمرينات +11 بر بر امتيازات آزمون كام آمرام

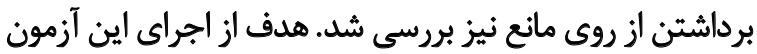

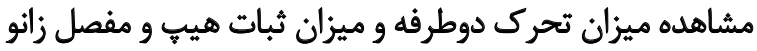

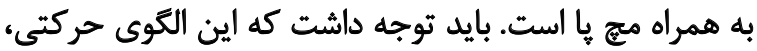

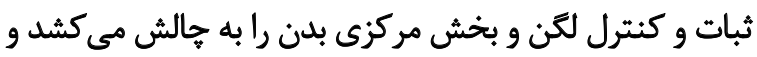

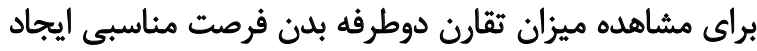

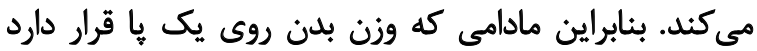

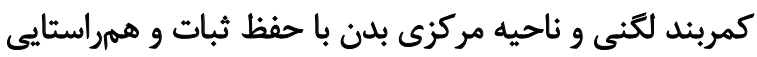

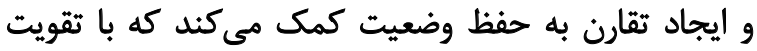

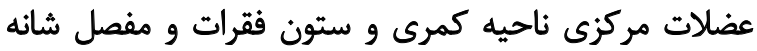

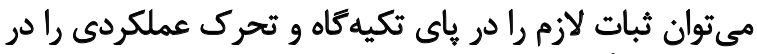

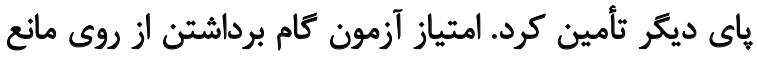

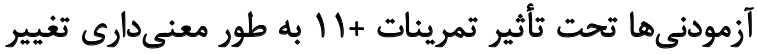

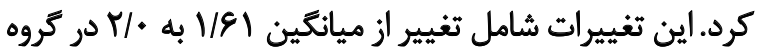

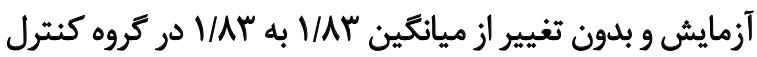

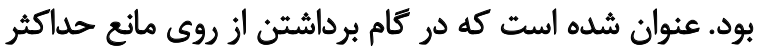

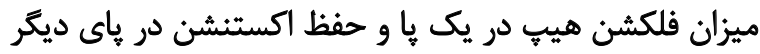

هماهنكّى عصبى ـ عضلانى مناسب و نيروى انتقال در نيمتنه است

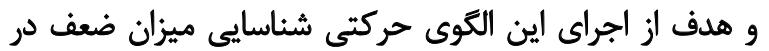

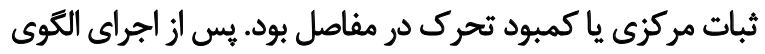

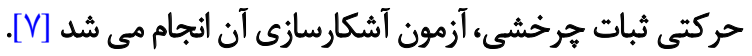
يروتكل تمرينى اعمال شده در اين تحقيق، +11 اليا برنامه

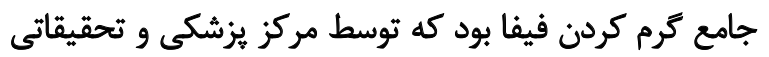

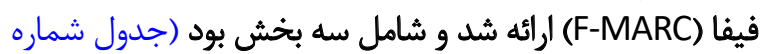

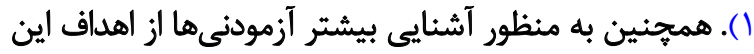

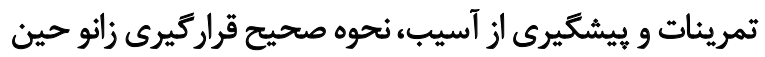

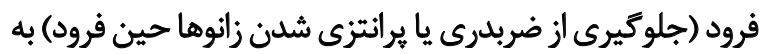
طور كامل شرح داده شد.

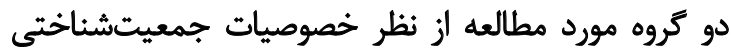

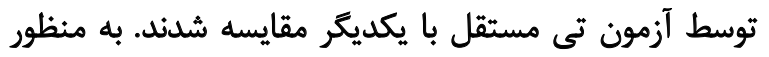

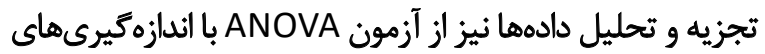

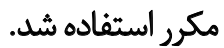
ثنايج

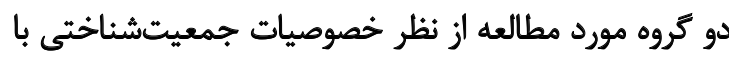

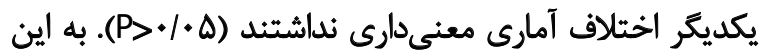

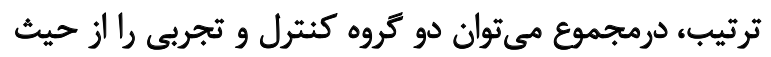

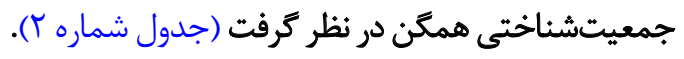
در بررسى اثر كروهبندى بر امتياز آزمون ديب اسكات، تأثيرات

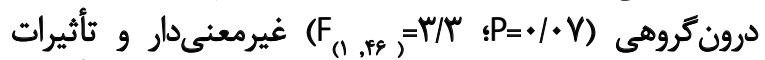

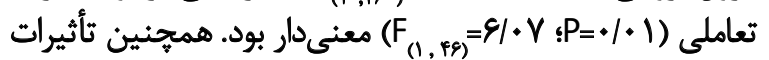

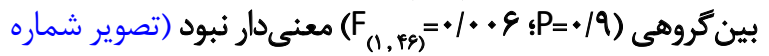

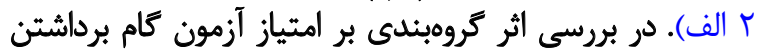

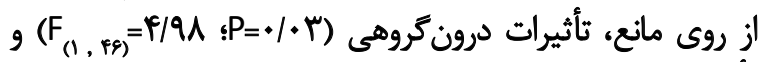

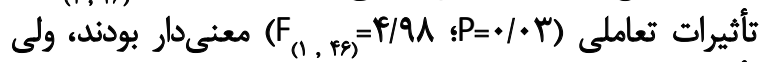

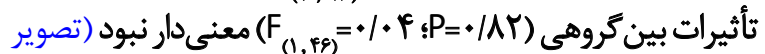

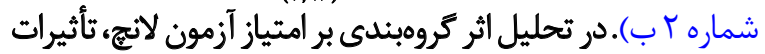

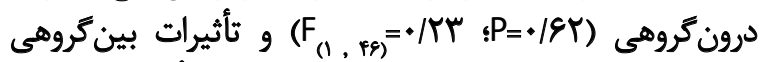

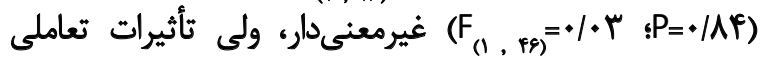

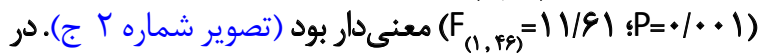

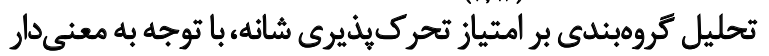

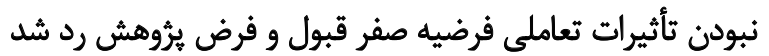

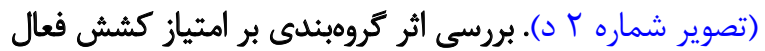

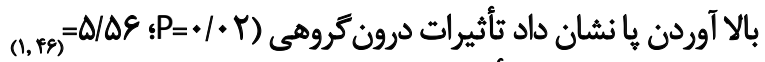

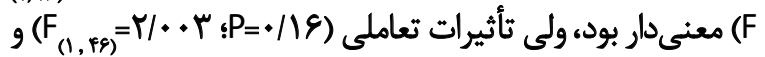

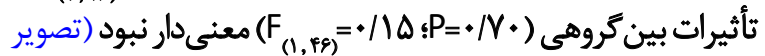

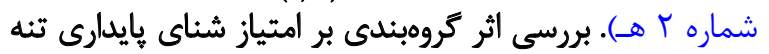

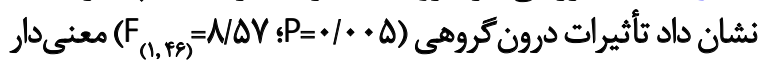

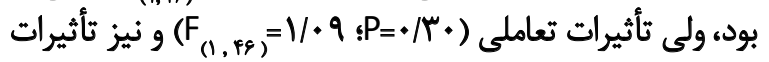

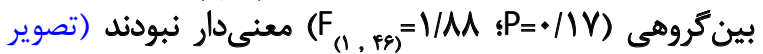




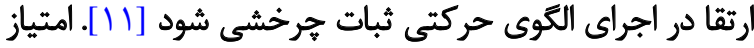

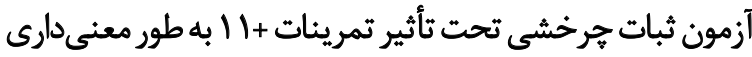

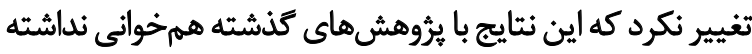

\section{نتيجلكيرى نهايى}

نتيجه كلى يافتههاى اين بروهش بيانكر آن است كه تمرينات

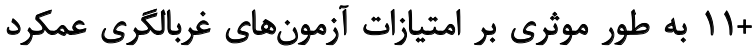

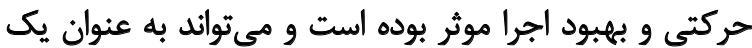

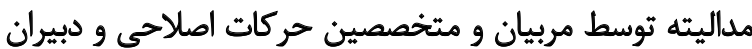

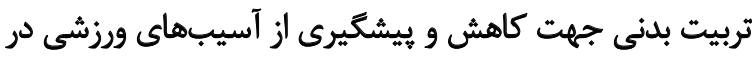

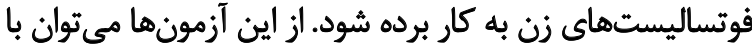

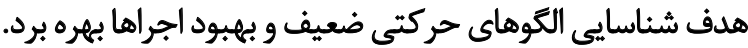

ماحظات اخلاقى

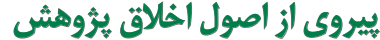

در اين مطالعه، يَ از مشاوره با داوطلبان از تمامى آزمودنى هائا

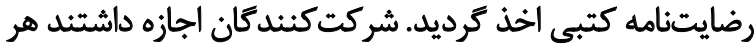

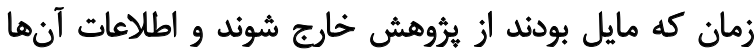
محرمانه نكَه داشته شد.

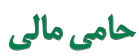

اين مقاله مستخرج از زاياننامه كارشناسى ارشد نويسنده

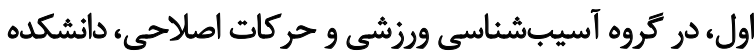

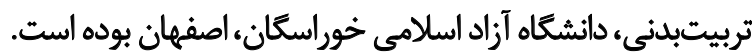

$$
\text { مشاركت نويسند مَان }
$$

كنارش متن مقاله و استخراج اطلاعات و تحليل دادهها: اكرم

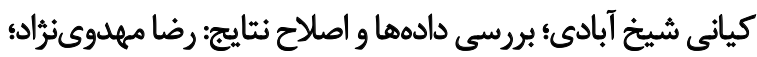

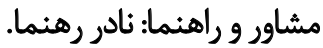

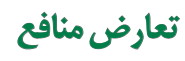

بنابر اظهار نويسندكان اين مقاله تعارض منافع ندارد.

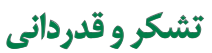

بدينوسيله از معاونت يرؤهشى دانشكاه خوراسكان (اصفهان)

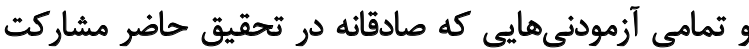
داشتند تشكر و قدردانى مى إنود.
وجود دارد و بايد به عدم تقارن نسبى در مفصل هيت و ميزان

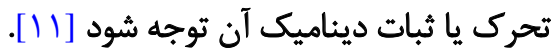

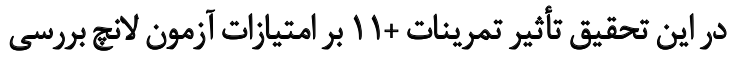

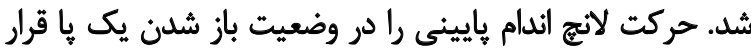

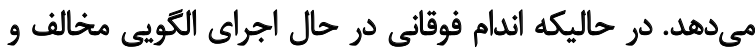

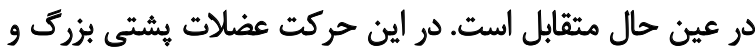

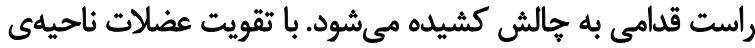

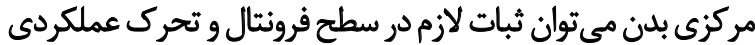

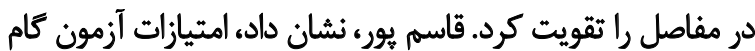

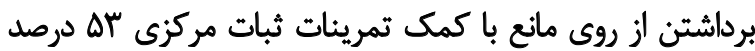

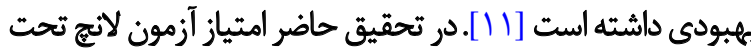

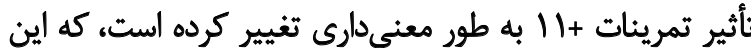

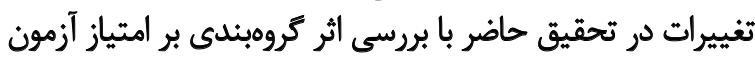

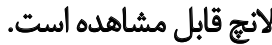
در اين تحقيق تأثير تمرينات +11 بر امتيازات آزمون تحرك دئ

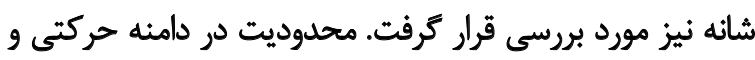

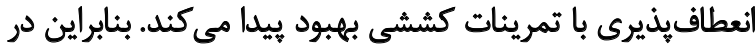

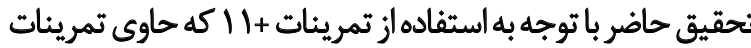

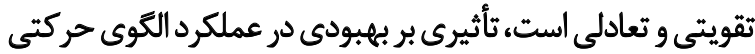

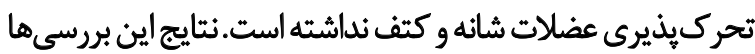

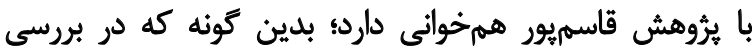

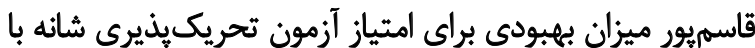
تمرينات ثابت مركزى، ها الدرصد است [11] اثر تمرينات +11 بر امتيازات آزمون كشش فعال يا در تحقيق

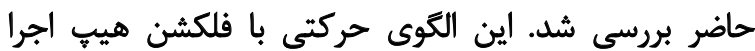

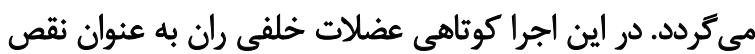

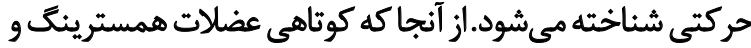

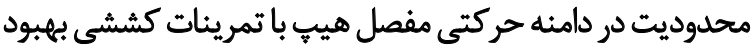

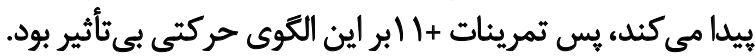

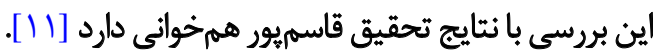
در الكوى شناى پايدارى تنه، محدوديت تحرك در مفصل

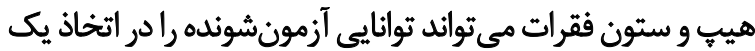

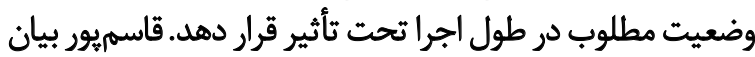

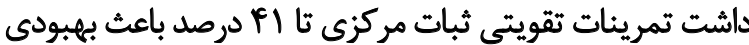

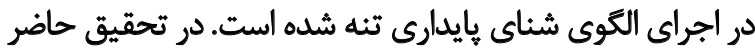

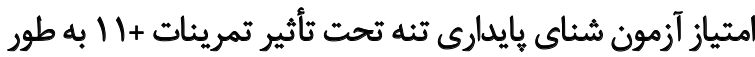

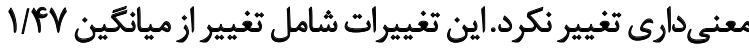

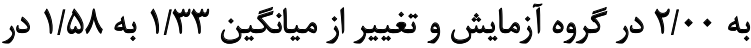
كروه كنترل بود كه بانتايج تحقيقات كذشته همرخوانى ندارد. هدف از اجراى الكوى حركتى ثبات خرخشى، شناسايى ميزان

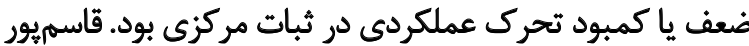

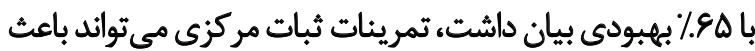




\section{References}

[1] Harkness EF, Macfarlane GJ, Silman AJ, McBeth J. Is musculoskeletal pain more common now than 40 years ago?: Two population-based cross-sectional studies. Rheumatology (Oxford). 2005; 44(7):890-5. [DOI:10.1093/rheumatology/keh599] [PMID]

[2] Valderon M, Gray A, Worsfold PR, Twist C. The reliability of Functional Movement Screening (FMS) and in-season changes in physical function and performance among elite rugby league players. J Strength Cond Res. 2016; 30(4):910-8. [DOI: 10.1519/JSC.0000000000000270]

[3] O'Connor FG, Deuster PA, Davis J, Pappas CG, Knapik JJ, Functional movement: Predicting injuries in officer candidates. Med Sci Sports Exerc. 2011; 43(12):2224-30 [DOI:10.1249/MSS.0b013e318223522d] [PMID]

[4] Hootman JM, Dick R, Agel J. Epidemiology of collegiate injuries for 15 sports: Summary and recommendations for injury prevention initiatives. J Athl Train. 2007; 42(2):311-9. [PMCID]

[5] Imwalle LE, Myer GD, Ford KR, Hewett TE. Relationship between hip and knee kinematics in athletic women during cutting maneuvers: A possible link to noncontact anterior cruciate ligament injury and prevention. J Strength Cond Res. 2009; 23(8):2223-30. [DOI:10.1519/ JSC.0b013e3181bc1a02] [PMID] [PMCID]

[6] Hewett TE, Ford KR, Hoogenboom BJ, Myer GD. Understanding and preventing $\mathrm{ACL}$ injuries: Current biomechanical and epidemiologic consideration-update 2010. N Am J Sports Phys Ther. 2010; 5(4):234-51. [PMCID]

[7] Cook G, Burton L, Hoogenboom B. Pre participation screening: The use of fundamental movements as an assessment of function-part 1 . Am J Sports Phys Ther. 2006; 1(2):62-72. [PMCID]

[8] Chorba RS, Chorba DJ, Bouillon LE, Overmyer CA, Landis JA. Use of a functional movement screening tool to determine injury risk in female collegiate athletes. N Am J Sports Phys Ther. 2010; 5(2):47-54. [PMCID]

[9] Sorenson EA. Functional movement screen as predictors of injury in high school basketball athletes [PhD. Dissertation]. Oregon: University of Oregon; 2009. http://hdl.handle.net/1794/10594

[10] Shojaedin SS, Letafatkar A, Hadadnezhad M, Dehkhoda MR. Relationship between functional movement screening score and history of injury and identifying the predictive value of the FMS for injury. Int J Inj Contr Saf Promot. 2014; 21(4):355-60. [DOI:10.1080/17457300.2013.8 33942] [PMID]

[11] Qasempour Kh. [The effect of 8 weeks of central stability exercises on the scores of motor performance screening tests in adolescents, Effect of 8 weeks of central stability exercises on the scores of functional movement screening (Persian)] [MSc. Thesis]. Isfahan: Islamic azad university; 2015

[12] Daneshjoo D, Mokhtar AH, Rahnama N, Yusof A. 2012, THe Effects of the 11+ and HarmoKnee warm-up programs on performance measures in professional soccer players. J Sports Sci Med. 2013; 12(3):489-96. [PMCID] [PMID] 